\title{
Synthesis of acridone-naphthylamine derivative and its thermally- activated delayed fluorescence studies for application in OLEDs
}

\author{
ANKUR A AWASTHI $^{\mathrm{a}}$, NIKITA GUPTA ${ }^{\mathrm{a}}$, QAMAR T SIDDIQUI ${ }^{\mathrm{a}, \mathrm{b}}$, PRADNYA PARAB $^{\mathrm{c}}$, \\ DIPAK K PALIT ${ }^{\mathrm{a}}$, SANGITA BOSE $^{\mathrm{c}, *}$ and NEERAJ AGARWAL ${ }^{\mathrm{a}, *}$ \\ ${ }^{a}$ School of Chemical Sciences, UM-DAE Centre for Excellence in Basic Sciences, University of Mumbai, \\ Kalina, Santacruz (E), Mumbai 400 098, Maharashtra, India \\ ${ }^{\mathrm{b}}$ Department of Chemistry, Aligarh Muslim University, Aligarh, Uttar Pradesh, India \\ ${ }^{\mathrm{c}}$ School of Physical Sciences, UM-DAE Centre for Excellence in Basic Sciences, University of Mumbai, \\ Kalina, Santacruz (E), Mumbai 400 098, Maharashtra, India \\ E-mail: sangita@cbs.ac.in; na@cbs.ac.in
}

MS received 30 March 2019; revised 19 May 2019; accepted 22 May 2019

\begin{abstract}
Acridone (acceptor) and naphthylamine (donor) based Donor-Acceptor-Donor (D-A-D) compound (1) was synthesised, characterised and its thermally-activated delayed fluorescence (TADF) properties were studied in detail. Compound $\mathbf{1}$ is fluorescent and emits in the green region $(550 \mathrm{~nm})$. The energy gap between the ground and the lowest excited singlet $\left(\mathrm{S}_{1}\right)$ state is estimated to be $2.55 \mathrm{eV}$. The energy gap between the CT singlet and triplet states $\left(\Delta \mathrm{E}_{\mathrm{ST}}\right)$ was found to be $\sim 0.3 \mathrm{eV}$. Small $\Delta \mathrm{E}_{\mathrm{S} 1-\mathrm{T} 1}$ is one of the important criteria for TADF to take place in a molecule and thus detailed photophysics has been studied. Transient lifetime measurements showed an increase in the fluorescence lifetime $(\tau)$ on purging with $\mathrm{N}_{2}$, as compared with that in air-saturated solution, indicating the involvement of the triplet state in emission. Emission at $550 \mathrm{~nm}$ was also observed with a delay of $100 \mu$ s which corresponded to the delayed fluorescence in $\mathbf{1}$. The lifetime of TADF was found to be $176 \mu \mathrm{s}$. Applications of TADF materials in organic lightemitting devices (OLEDs) has gotten attention as TADF materials utilise the triplet excitons which helps in increasing internal quantum efficiency of device. Air-saturated based on 1 were fabricated and their intensity was found to be nearly as high as $17,000 \mathrm{Cd} / \mathrm{m}^{2}$ at $25 \mathrm{~mA} / \mathrm{cm}^{2}$ which was comparable to many of the known TADF emitters.
\end{abstract}

Keywords. Acridone; thermally activated delayed fluorescence; organic light-emitting devices.

\section{Introduction}

As the demand for cost-competitive and renewable energy options grows, considerable attention is being placed on the development of new materials for low energy consuming devices. Organic semiconductors offer a range of low-cost device applications, for example, large-area displays, organic light-emitting devices (OLED) and solar cell. ${ }^{1-8}$ Organic chemistry offers a great advantage in designing a large variety of materials of similar chemical structures with tunable photophysical properties by derivatization and this has made possible the development of more efficient organic semiconductors for solid-state devices including OLEDs. Typically, under electrical excitation, fluorescent molecule generates up to $25 \%$ singlet and $75 \%$ non-emissive triplets excitons which results in only $25 \%$ internal quantum efficiency (IQE). The non-emissive triplet excitons are deactivated via nonradiative processes generating heat. ${ }^{9-12}$ Thus, significant research is being pursued to utilize the triplet excitons improving the IQE at room temperature. Electroluminescence in OLEDs arises from the singlet state in fluorescent materials, while that is from the triplet state in phosphorescent emitters. It has been shown that $100 \%$ internal efficiency of OLEDs can be obtained when noble metal (e.g., Pd, Pt, Ir, etc.) based phosphorescent materials are used as an emitter. ${ }^{13,14}$

*For correspondence

Electronic supplementary material: The online version of this article (https://doi.org/10.1007/s12039-019-1667-9) contains supplementary material, which is available to authorized users. 
Development of efficient and cost-effective noble metal-containing phosphorescent materials for the entire visible region is still challenging and limits their commercial production. Recently, it has been shown that metal-free organic molecules capable of showing thermally activated delayed fluorescence (TADF) can be used to access both the singlet and triplet excitons for emission. To enhance the efficiency of OLEDs, TADF enabled molecules are considered as alternative to phosphors. ${ }^{8,10,15-19}$ Materials having small energy difference between the lowest singlet $\left(\mathrm{S}_{1}\right)$ and triplet $\left(\mathrm{T}_{1}\right)$ excited states promote reverse intersystem crossing (RISC) at room temperature and thus populate the $S_{1}$ state. Such emission from $S_{1}$ as a result of RISC is reported as thermally activated delayed fluorescence (TADF). TADF capable materials have potential to populate the singlet by using the triplet states, therefore they are considered as better emissive materials for OLEDs. ${ }^{20,21}$ It is envisaged that with TADF materials, $100 \%$ internal efficiency (theoretically) can be obtained in OLEDs as is the case with phosphors. High fluorescence quantum yield, short lifetime of the $\mathrm{S}_{1}$ state and small $\Delta \mathrm{E}_{\mathrm{ST}}(\sim 0.3 \mathrm{eV})$ are the important criteria for organic molecules to act as TADF emitter. The main challenge in designing a TADF molecule is to ensure a small energy gap between the lowest excited singlet and triplet states $\left(\Delta \mathrm{E}_{\mathrm{ST}}\right)$. TADF molecules, in which an electron donor (D) and acceptor (A) moieties are covalently linked, have been reported to have small $\Delta \mathrm{E}_{\mathrm{ST}}$. Such materials show intramolecular charge transfer (ICT) character, which results in very small overlap between the highest occupied molecular orbital (HOMO) and lowest unoccupied molecular orbital (LUMO). Small or no overlap between the HOMO and LUMO leads to decrease in electronic exchange energy, thus helping to obtain small energy gap between the singlet and triplet states. $^{22}$

Push-pull molecules based on anthraquinone, acridone, quinacridone, etc., having singlet and triplet states with strong charge-transfer character, have been reported to possess small $\Delta \mathrm{E}_{\mathrm{ST}}$ and were explored for TADF properties. ${ }^{16,23-26}$ Recently, we reported the aromatic amine (cyclic as well as acyclic) substituted acridone derivatives and their photophysical properties. ${ }^{16,27}$ In our earlier results carbazole (cyclic amine) substituted acridone derivatives showed TADF properties. In this article, we report the design and synthesis of acridone derivative substituted with acyclic bulky $2,2^{\prime}$-dinaphthylamine, $\left(2,7\right.$-bis- $\left(N, N^{\prime}\right.$-di2-naphthyl)-10-methylacridone, 1). In compound $\mathbf{1}$, acridone core was chosen as electron acceptor and dinaphthylamine as electron donor. Our earlier results with acyclic amine substituted acridone showed promising results for organic electronics materials. We further modified the design and chose naphthyl amine as a bulky substituent which can restrict the rotation of amine and is expected to bring non-planarity in the molecule. Non-planar materials having strong electron acceptor and donating moieties are expected to show charge transfer property, one of the important criterion for TADF materials. Photophysical properties of 1 were studied in detail which shows that it possesses TADF character. OLEDs were also fabricated which showed green emission with a light intensity of $17,000 \mathrm{Cd} / \mathrm{m}^{2}$ at a current density of $25 \mathrm{~mA} / \mathrm{cm}^{2}$.

\section{Experimental}

\subsection{Materials and instruments}

Reagents and solvents were purchased from Sigma Aldrich or S. D. Fine Chemicals and used as received. Solvents were dried, wherever anhydrous solvents were required. For absorption and emission, solvents used were of spectroscopic grades. ${ }^{1} \mathrm{H}$ NMR were recorded on a Varian $600 \mathrm{MHz}$ spectrometer. Mass spectra was recorded using Bruker MALDI-TOF. Cyclic Voltammetry was performed on $\mathrm{CH}$ Instrument (620D). Three electrode cell was employed with a glassy carbon working electrode, $\mathrm{Ag} / \mathrm{AgCl}$ (non-aqueous) reference electrode and $\mathrm{Pt}$ wire counter electrode. The measurements were performed in anhydrous acetonitrile with tetrabutylammonium hexafluorophosphate as supporting electrolyte at a scan rate of $100 \mathrm{mV} / \mathrm{s}$. UV-visible spectra were recorded using a Shimadzu 1800 from $800 \mathrm{~nm}$ to $200 \mathrm{~nm}$. Steady-state fluorescence spectrophotometer were recorded on a Horiba Fluoromax-4 spectrofluorometer, with sample excited at $410 \mathrm{~nm}$. A diode laser-based time-correlated singlephoton counting (TCSPC) spectrometer (IBH, U.K.) was used to obtain the excited-state lifetimes. The sample was excited using a $406 \mathrm{~nm}$ laser to obtain the lifetime. TADF lifetime was obtained on an Agilent spectrofluorometer, with emission recorded after a delay time (100-400 $\mu$ s). Emission peak intensity was plotted against the delay time and fitted using equation $\mathrm{I}=\mathrm{I}_{\mathrm{o}} \mathrm{e}^{-\mathrm{t} / \tau}$. Wherever required, deconvolution was performed on origin 9 software using nonlinear curve fit operation. Gaussian function and Levenberg-Marquardt Iteration Algorithm was used to fit the multi peaks. For OLED fabrication, films were grown by spin coating (using a Holmarc HO-TH-05 spin coater) at $2000 \mathrm{rpm}$ for $40 \mathrm{~s}$. Vacuum deposition was carried out at a base pressure of $2 \times 10^{-6}$ mbar. ITO coated substrate (15-25 $\Omega / \mathrm{sq}$, Sigma Aldrich) was etched into desired pattern. Substrate cleaning was done with soap solution, propanol and trichloroethylene (TCE) vapours in stepwise manner. UV treatment was given before using 
them in device fabrication. The organic layer of $\mathbf{1}$ was thermally evaporated in vacuum giving a film of $\sim 50 \mathrm{~nm}$ over a layer of PEDOT:PSS which was spin-coated ( $\sim 50 \mathrm{~nm}$ thick) and a layer of $N, N^{\prime}$-Bis(naphthalen-1-yl)$N, N^{\prime}$-bis(phenyl)benzidine (NPD; $\sim 40 \mathrm{~nm}$ ). The thickness of PEDOT:PSS and NPD were measured by surface profilometer (DEKTAK). NPD, bathophenanthroline (Bphen; $\sim 25 \mathrm{~nm}), \operatorname{LiF}(\sim 1 \mathrm{~nm})$, and $\mathrm{Al}(\sim 160 \mathrm{~nm})$ were all thermally evaporated. The geometry of the most optimized device was ITO/(PEDOT:PSS/ NPD/1/Bphen/ $\mathrm{LiF} / \mathrm{Al}$.

\subsection{Synthesis}

2,7-Dibromo-10-methylacridone was synthesized according to literature.

1: Compound 1 was synthesized by Buchwald-Hartwig amination. 2,7-Dibromo-10-methylacridone $\quad(0.075 \mathrm{~g}$, $0.20 \mathrm{mmol})$ and $2,2^{\prime}$-dinaphthylamine $(0.13 \mathrm{~g}, 0.50 \mathrm{mmol})$ were dissolved in anhydrous toluene $(20 \mathrm{~mL})$ under argon atmosphere. $\left[\mathrm{Pd}_{2}(\mathrm{dba})_{3}\right]$ (5-8 mol\%), 2-dicyclohexylphoshpino-2', $6^{\prime}$-dimethylbiphenyl (SPhos) (10-15 mol\%) and sodium-t-butoxide $(3.1 \mathrm{mmol})$ were added to the reaction mixture. The reaction mixture was thoroughly stirred under argon atmosphere while the temperature was slowly raised to $100{ }^{\circ} \mathrm{C}$ and refluxed for $18 \mathrm{~h}$. Product was extracted using dicholoromethane (DCM) and organic layers were washed with water. Crude mixture was purified through column chromatography using 50\% DCM in Hexane. Yield: $8 \%{ }^{1} \mathrm{H}$ $\mathrm{NMR}\left(\mathrm{CDCl}_{3}, 600 \mathrm{MHz}, \delta \mathrm{ppm}\right): 8.30(\mathrm{~d}, 2 \mathrm{H}, J=2.4 \mathrm{~Hz})$, $7.74(\mathrm{~m}, 8 \mathrm{H}) ; 7.63(\mathrm{~d}, 1 \mathrm{H}, J=3.0 \mathrm{~Hz}) ; 7.61(\mathrm{~d}, 1 \mathrm{H}$, $J=2.4 \mathrm{~Hz}) ; 7.56(\mathrm{~s}, 2 \mathrm{H}) ; 7.55(\mathrm{~s}, 2 \mathrm{H}) ; 7.53(\mathrm{~s}, 1 \mathrm{H}) ; 7.51(\mathrm{~s}$, $1 \mathrm{H}) ; 7.44$ (d, $4 \mathrm{H} J=2.4 \mathrm{~Hz}) ; 7.39$ (d, $1 \mathrm{H}, J=1.8 \mathrm{~Hz}) ; 7.38$ $(\mathrm{d}, 2 \mathrm{H}, J=1.2 \mathrm{~Hz}) ; 7.37(\mathrm{~s}, 1 \mathrm{H}, J=1.6 \mathrm{~Hz}) ; 7.36(\mathrm{~s}, 1 \mathrm{H}$, $J=1.6 \mathrm{~Hz}) ; \quad 7.35(\mathrm{~d}, \quad 2 \mathrm{H}, \quad J=0.6 \mathrm{~Hz}) ; 7.33 \quad(\mathrm{~d}, 3 \mathrm{H}$, $J=2.4 \mathrm{~Hz}) ; 7.32$ (d, 2H, $J=1.8 \mathrm{~Hz}) ; 3.95$ (s, 3H). MALDITOF: $\mathrm{m} / \mathrm{z}[\mathrm{M}]^{+}$calcd. $\mathrm{C}_{54} \mathrm{H}_{37} \mathrm{~N}_{3} \mathrm{O}, 743.89$; found: 742.42 .

\section{Results and Discussions}

\subsection{Synthesis}

Compound 1 was synthesized in three steps, starting from acridone, as shown in Scheme 1. For methylation, $9(10 \mathrm{H})$-acridone was reacted with iodomethane in the presence of $\mathrm{NaH}$. A light-yellow solid product, methylacridone, was further brominated using molecular bromine in acetic acid. Brominated acridone was then dried, purified and identified as 2,7-dibromo-10methylacridone as yellow solid. ${ }^{27}$ The amination of 2,7-dibromo-10-methylacridone and 2,2'-dinaphthylamine was carried using B-H amination as mentioned in the experimental section. Pure 1 was obtained in $\sim 8 \%$ yield. Compound $\mathbf{1}$ was characterised by ${ }^{1} \mathrm{H}-$ NMR and MALDI-TOF (Supplementary Information).

\subsection{Photophysical studies}

Absorption and emission properties of $\mathbf{1}$ have been studied in acetonitrile and spectra are shown in Figure 1 and tabulated in Table 1. The absorption spectrum shows multiple bands. Two prominent bands with maxima at $\sim 260$ and $345 \mathrm{~nm}$ are the characteristics of $2,2^{\prime}$-dinaphthylamine. A broad shoulder in the $400-500 \mathrm{~nm}$ region is assigned to the acridone moiety. The emission spectrum of compound $\mathbf{1}$ shows a maximum at $550 \mathrm{~nm}$. The energy gap between the ground state $\left(\mathrm{S}_{0}\right)$ and the first excited singlet state $\left(\mathrm{S}_{1}\right)$ is determined from the intersection of emission and excited spectra, which corresponds to $2.55 \mathrm{eV}$ (Figure S3, Supplementary Information). The fluorescence quantum yields $\left(\phi_{\mathrm{F}}\right)$ of $\mathbf{1}$ was determined by using a comparative method using curcumin in acetonitrile as<smiles>O=c1c2ccccc2[nH]c2ccccc12</smiles>

i) $\mathrm{NaH}$ at $0^{\circ} \mathrm{C}, 30 \mathrm{~min}$

ii)

$\underset{\text { dry DMF }}{60^{\circ} \mathrm{C}, \mathrm{CH}_{3} \mathrm{I}} \longrightarrow$<smiles>Cn1c2ccccc2c(=O)c2ccccc21</smiles>

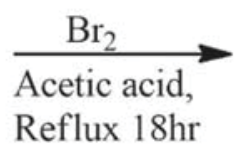<smiles>Cn1c2ccc(Br)cc2c(=O)c2cc(Br)ccc21</smiles><smiles>Cn1c2ccc(Br)cc2c(=O)c2cc(Br)ccc21</smiles>

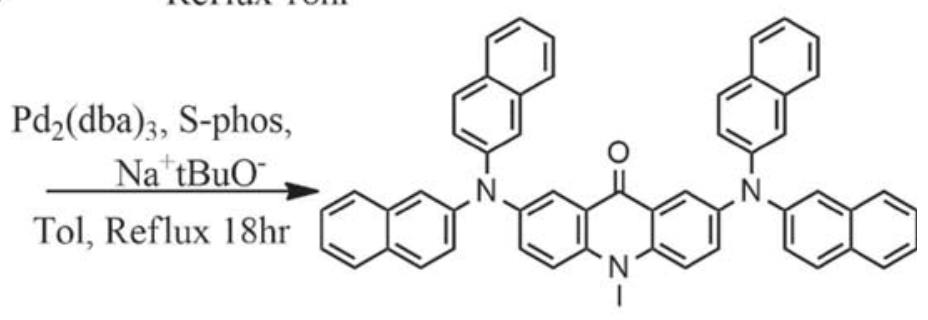

Scheme 1. Synthesis of acridone-dinaphthylamine derivative, $\mathbf{1}$. 


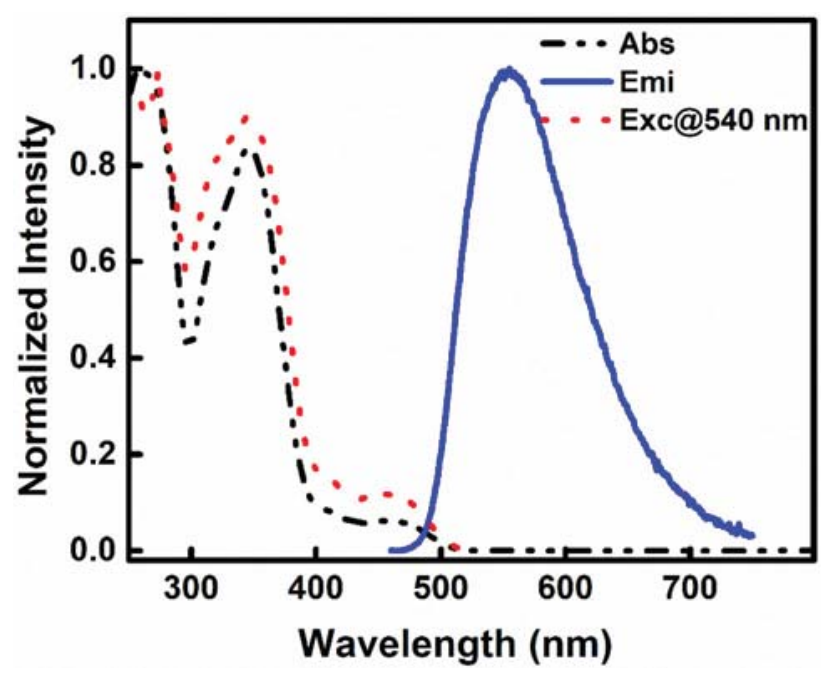

Figure 1. Absorption, emission and excitation spectra of the compound $\mathbf{1}$ in acetonitrile.

the standard $(\mathrm{Q} . \mathrm{Y} .=0.075) .{ }^{28}$ Fluorescence quantum yield was found to be 0.09 for $\mathbf{1}$.

In acetonitrile, the broad emission band suggests ICT character of the $S_{1}$ state. ICT character of 1 was further investigated by solvatochromic study. The absorption and emission spectra of $\mathbf{1}$ were recorded in various solvents of different polarities and they have been shown in Figure S4 and S5, respectively (Supplementary Information). While the absorption characteristics (such as width and maximum of the spectrum) of $\mathbf{1}$, which is a D-A-D type molecule having 2,2'-dinaphthylamine as the electron donor and 10-methylacridone as the electron acceptor moieties, are independent on solvent polarity, characteristics of the emission spectrum show a strong polarity dependence. The emission spectrum recorded in hexane shows a maximum at $490 \mathrm{~nm}$ and a shoulder at $525 \mathrm{~nm}$, which could be assigned to the vibronic features. ${ }^{29}$ The width of the emission spectrum increases and the emission maximum displays larger bathochromic shift as the polarity of the medium increases. The shift is as large as about $25 \mathrm{~nm}$ in acetonitrile as compared to that in hexane. These observations confirm the prediction about the strong ICT character of the excited state of $\mathbf{1}$.

To understand the possibility of delayed fluorescence in $\mathbf{1}$, it is desirable to know the photophysical

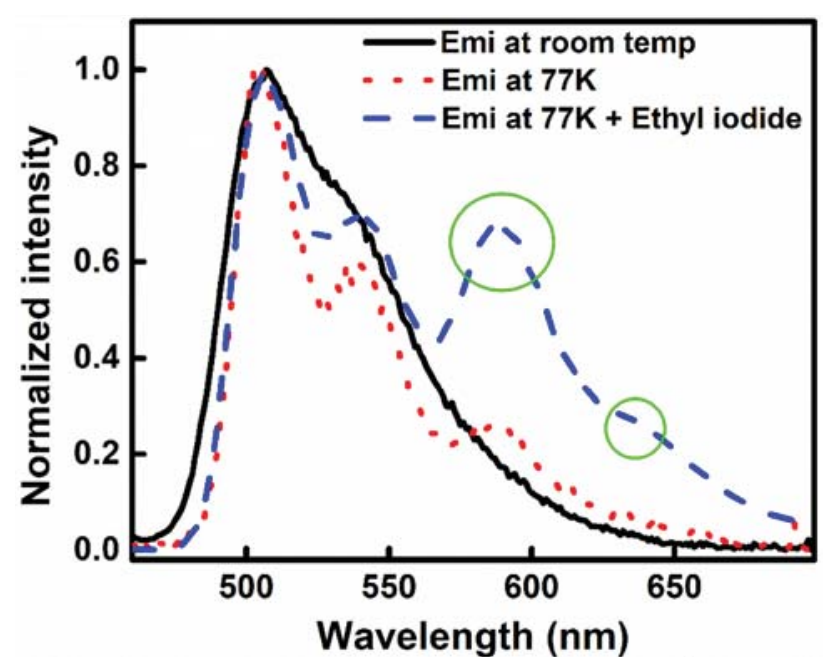

Figure 2. Emission spectra of $\mathbf{1}$ in methyl THF at room temperature (black), at $77 \mathrm{~K}$ (red) and at $77 \mathrm{~K}$ with ethyl iodide (blue).

properties of the phosphorescent or the triplet energy state of the molecule. Due to the long lifetime (in the range of a few $\mu$ s to ms) of the triplet state, the triplet state emission in liquid solution at room temperature is efficiently quenched via various non-radiative processes, for example, intermolecular collisional quenching, vibrational relaxations, etc. ${ }^{30,31}$ To eliminate the possibility of the triplet state emission quenching, emission spectrum of $\mathbf{1}$ was recorded in THF solvent matrix at $77 \mathrm{~K}$. This emission spectrum shows two additional bands with the emission maxima at 590 and $635 \mathrm{~nm}$ in addition to those due to fluorescence emission at 500 and $530 \mathrm{~nm}$, which could be considered to be arising from the triplet state (phosphorescence).

The intensity of the phosphorescence band (triplet emission) is found to be smaller as compared to that of singlet emission. Addition of ethyl iodide $(\sim 10 \% \mathrm{v} / \mathrm{v})$ to the solvent matrix increases the intensities of phosphorescence bands by several folds (Figure 2) due to heavy atom effect. Heavy atoms (I, Pt, Pd, Ru, etc.) are known to enhance the triplet state population. ${ }^{16}$ Thereby, it confirms that emission bands at 590 and $635 \mathrm{~nm}$ at low temperature are arising from the triplet state(s). The emission spectrum recorded at $77 \mathrm{~K}$ in the THF solvent matrix containing ethyl iodide was

Table 1. Photophysical data of $\mathbf{1}$.

\begin{tabular}{ccccccc}
\hline Comp. & ${ }^{\mathrm{a}} \lambda_{\text {abs }} \mathrm{nm}$ & ${ }^{\mathrm{a}} \lambda_{\mathrm{em}}(\mathrm{nm})$ & ${ }^{\mathrm{a}} \phi_{\mathrm{F}}$ & ${ }^{\mathrm{a}} \tau_{\mathrm{PF}}(\mathrm{ns})$ & ${ }^{\mathrm{a}} \tau_{\mathrm{DF}}(\mu \mathrm{s})$ & ${ }^{\mathrm{b}} \Delta \mathrm{E}_{\mathrm{ST}} \mathrm{eV}$ \\
\hline 1 & $260,346,465$ & 550 & 0.09 & 11.7 (in air) & 70 (in air) & 0.3 \\
& & & & 18.6 (in $\left.\mathrm{N}_{2}\right)$ & 178 (in $\left.\mathrm{N}_{2}\right)$ & \\
\hline
\end{tabular}

${ }^{\mathrm{a}}$ In acetonitrile; ${ }^{\mathrm{b}}$ in Me-THF. 
deconvoluted to obtain the characteristic peaks corresponding to the singlet and triplet states (Figure S6, Supplementary Information). The energy levels (singlet and triplet) of 1 were estimated from the onset of fluorescence and phosphorescence peaks. The singlettriplet energy gap $\left(\Delta \mathrm{E}_{\mathrm{ST}}\right)$ was found to be $0.3 \mathrm{eV}$.

To obtain information on the relaxation of the excited states of $\mathbf{1}$, we studied the fluorescence lifetimes using TCSPC technique in solution. A picosecond (ps) diode laser of $375 \mathrm{~nm}$ and $75 \mathrm{ps}$ pulse duration was used for excitation. The TCSPC instrument has a time resolution of about 300 ps. Emission was monitored at $525 \mathrm{~nm}$. Fluorescence lifetimes were recovered by fitting the temporal profiles using iterative deconvolution method using multi-exponential fit functions. Fluorescence lifetimes of $\mathbf{1}$ were measured in a few solvents with air saturation as well as $\mathrm{N}_{2}$ purged (Figure 3). In all the cases, the fluorescence profiles could be fitted well with single exponential function and the lifetimes thus recovered are tabulated in Table 2, which suggests that lifetimes are nearly independent of the solvent polarity.
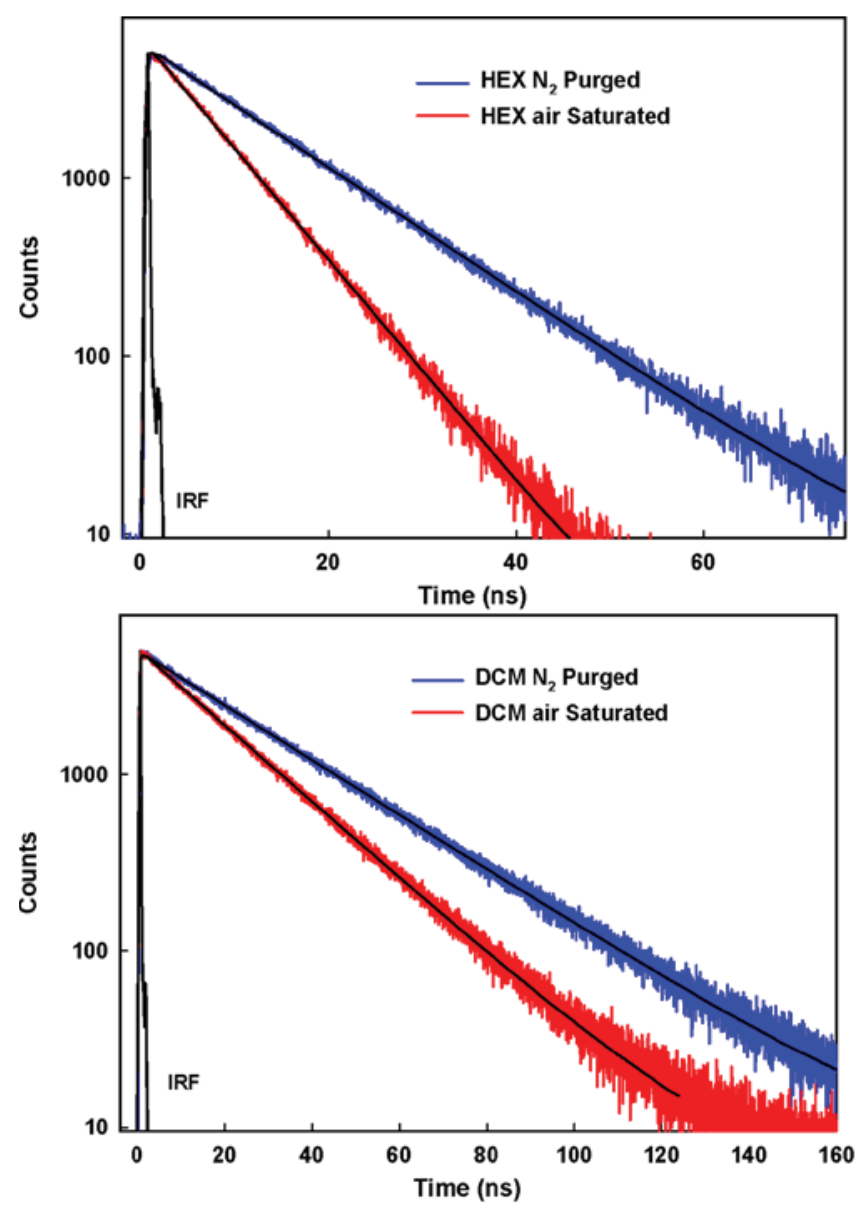

Earlier, it was proposed that a short TADF lifetime is beneficial to suppress triplet quenching processes like triplet-triplet annihilation, triplet-polaron annihilation, etc., thereby improving singlet fluorescence efficiency via TADF. ${ }^{32}$ The delayed fluorescence lifetime in TADF molecules is mostly in microseconds-milliseconds scale. ${ }^{10,33}$ Here, we have attempted to evaluate the TADF lifetime of compound $\mathbf{1}$ in acetonitrile solution. TADF process engages both the singlet and triplet states and thus the presence of molecular oxygen (air) and lifetime scale of these two states will affect the efficiency of the TADF emission. Fluorimetric studies were carried out to find out the TADF lifetime in an air-saturated as well as a nitrogen-purged atmosphere at room temperature. Here, we recorded emission spectra at various delay times ranging from $100 \mu$ s to $400 \mu$ s in air-saturated and nitrogen purged acetonitrile (Figures S7 and 8, Supplementary Information) and the peak intensity of emission (at $550 \mathrm{~nm}$ ) was plotted against delay time (Figure S9, Supplementary Information). Lifetime was calculated using the equation, $I=I_{o} e^{-t / \tau}$. Figure 4
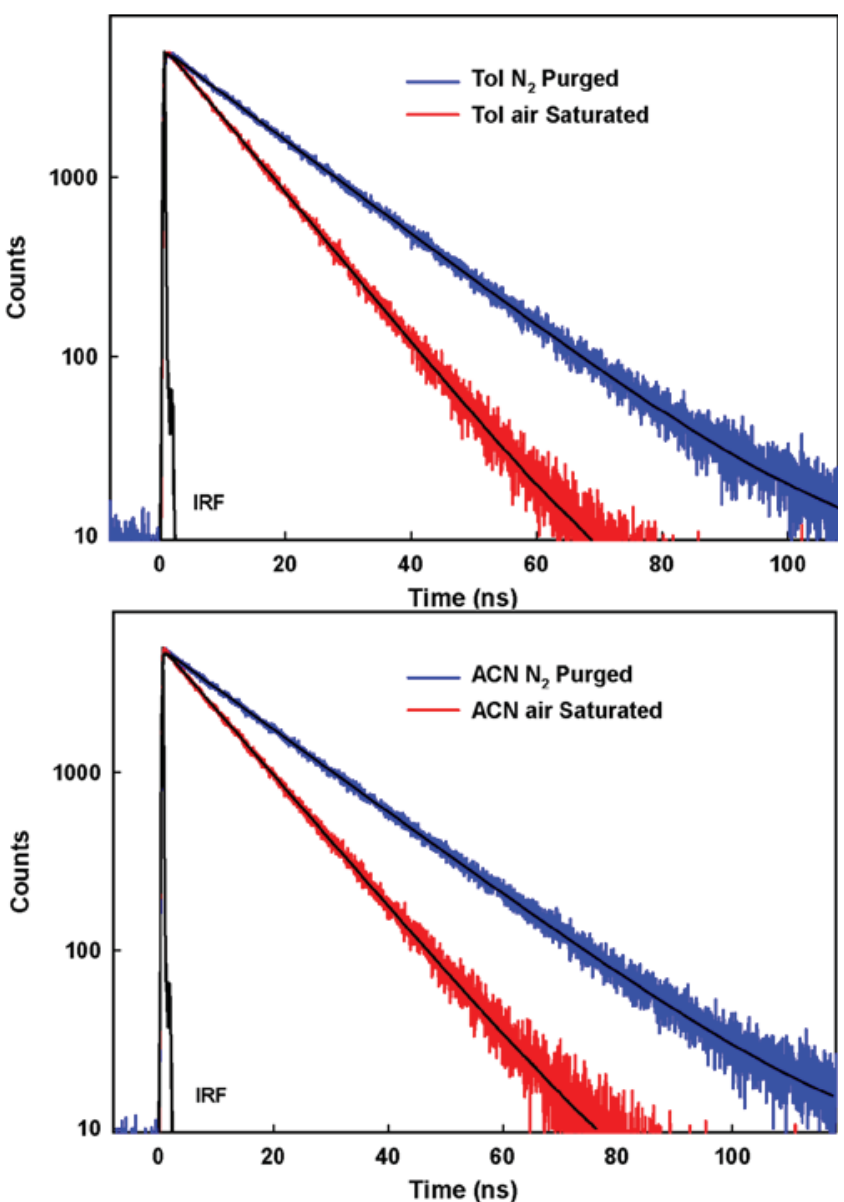

Figure 3. Fluorescence decay profile of $\mathbf{1}$ in different air saturated and nitrogen purged solvents. 
Table 2. Fluorescence lifetime (ns) data of $\mathbf{1}$ in different solvents.

\begin{tabular}{lcccc}
\hline & Acetonitrile & Hexane & Dichloromethane & Toluene \\
\hline Air-saturated & 11.7 & 6.9 & 19.9 & 10.3 \\
$\mathrm{~N}_{2}$ purged & 18.6 & 12.4 & 27.8 & 16.2 \\
\hline
\end{tabular}

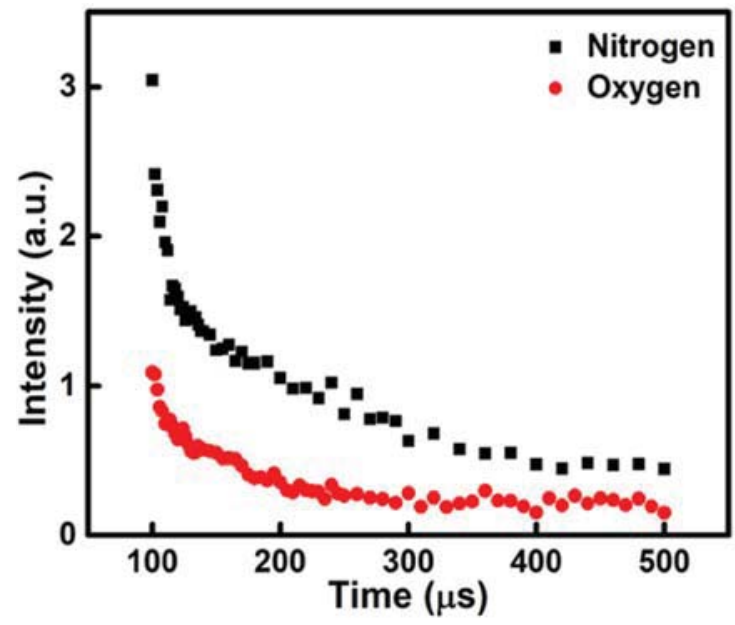

(a)

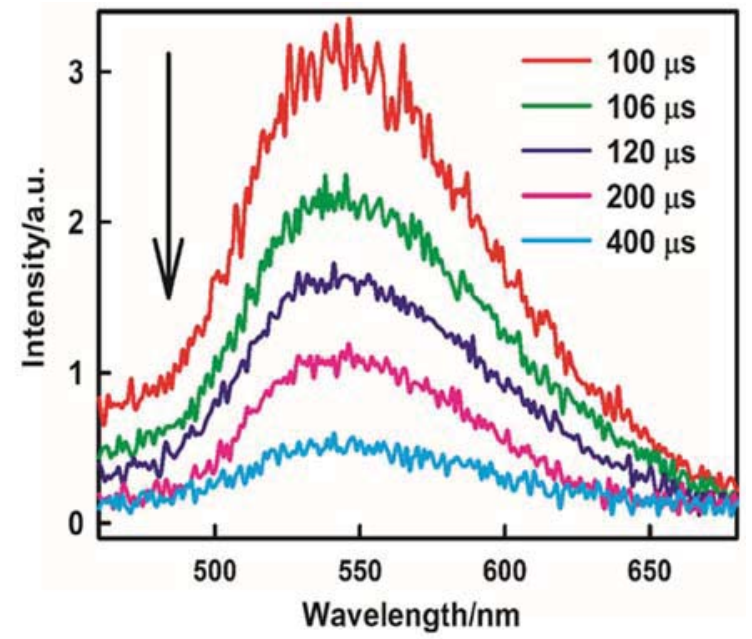

(b)

Figure 4. (a) Emission decay profile of $\mathbf{1}$ in acetonitrile at room temperature in air-saturated and nitrogen purged solvent. (b) Emission spectra of $\mathbf{1}$ recorded after various delays in a nitrogen-purged solvent.

shows the TADF emission and lifetimes of $\mathbf{1}$ in microseconds. Since delay time was kept much longer than $100 \mathrm{~ns}$, therefore, prompt fluorescence could be ruled out as prompt fluorescence lifetime is about $10 \mathrm{~ns}$ only. Also, the emission maximum $(550 \mathrm{~nm}$ in the same solvent) in the time-resolved emission spectra matches well with that observed in prompt fluorescence of $\mathbf{1}$. These observations clearly suggest that the emission process occurring at delay times longer than $100 \mu$ s time domain is due to the TADF process. In $\mathrm{N}_{2}$ purged solution, emission intensity was seen to increase and the lifetime $(\sim 178 \mu \mathrm{s})$ was also found to be longer than that $(\sim 70 \mu \mathrm{s})$ in air-saturated solution, thus confirming the participation of the triplet state in the emission process.

\subsection{Cyclic voltammetry}

Cyclic voltammetric studies were carried out to find out the HOMO and LUMO energy levels. $\mathrm{E}_{\mathrm{HOMO}}$ and $\mathrm{E}_{\text {LUMO }}$ play an important role in finding suitable electrodes and supporting organic materials while working on the device geometry. The highest occupied molecular orbital (HOMO) and lowest unoccupied molecular orbital (LUMO) energy levels of 1 were estimated using the oxidation potential and optical bandgap. The cyclic voltammetric method was used to find out the oxidation potential of $\mathbf{1}$. First oxidation peak was found at $0.65 \mathrm{~V}$. The HOMO energy levels of 1 were calculated using oxidation potentials, taking $\mathrm{Fc} / \mathrm{Fc}^{+}(0.33 \mathrm{~V})$ as a standard. ${ }^{34}$ Using oxidation potential, the HOMO energy of $\mathbf{1}$ is found to be roughly $\sim-5.4 \mathrm{eV}$. The optical gap was measured from the intersection of excitation and emission spectra and found to be $2.55 \mathrm{eV}$. Using $\mathrm{E}_{\mathrm{HOMO}}$ and optical gap, $\mathrm{E}_{\mathrm{LUMO}}$ was estimated at $\sim-2.85 \mathrm{eV}$.

\subsection{Organic light-emitting device and characterization}

To study the potential of $\mathbf{1}$ as an emissive layer in OLEDs, devices were fabricated and studied. The OLEDs were fabricated using $\mathbf{1}$ as a neat emitter without doping in any host material as the latter is believed to alter device properties. $\operatorname{Poly}(3,4-$ ethylenedioxythiophene) polystyrene sulfonate (PEDOT:PSS), a well-known hole injecting layer, is used in these studies. $\quad N, N^{\prime}$-di-(1-naphthyl)- $N, N^{\prime}$-diphenyl(1,1'-biphenyl)-4,4'-diamine (NPD) was used as hole transporting layer (HTL). The most optimized device 


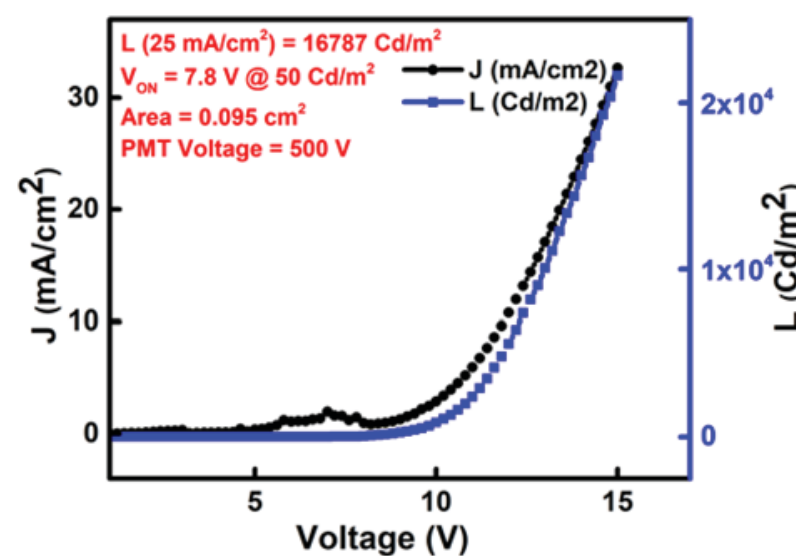

(a)

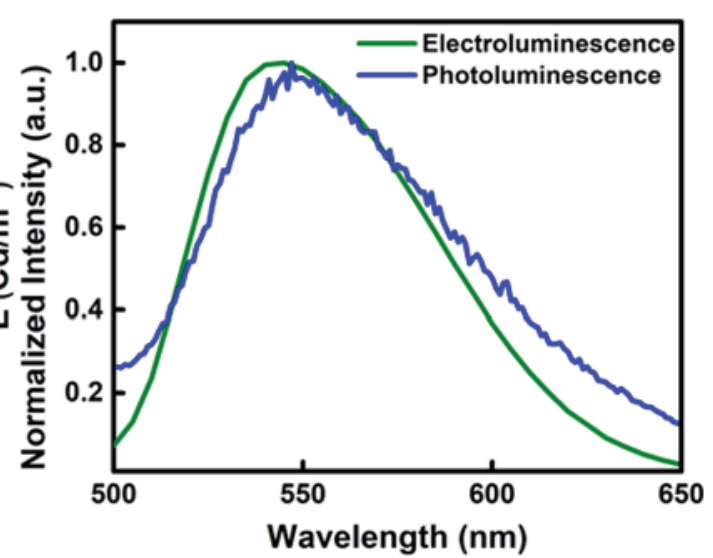

(b)

Figure 5. (a) J-V-L characteristics of the OLED device with the geometry ITO/PEDOT:PSS/NPD/1/BPhen/LiF/Al and (b) electroluminescence and photoluminescence (thin film) spectra.

structure used was ITO/PEDOT:PSS/NPD/1/BPhen/ LiF/Al. Typically, hole blocking layers (HBL) are most commonly placed after the emissive layer closer to the cathode to confine the carriers and excitons in emissive layer. 4,7-Diphenyl-1,10-phenanthroline (BPhen) was used as the hole blocking layer (HBL) in our devices. Electroluminance spectra of the devices showed the peak emission nearly at $550 \mathrm{~nm}$ and matches with the photoluminance of $\mathbf{1}$ (Figure 5). The turn-on voltage, $\mathrm{V}_{\mathrm{ON}}$ (voltage at which intensity is $50 \mathrm{Cd} / \mathrm{m}^{2}$ ) was found to be $7.8 \mathrm{~V}$. A high luminous intensity of $\sim 17000 \mathrm{Cd} / \mathrm{m}^{2}$ at a current density of $25 \mathrm{~mA} / \mathrm{cm}^{2}$ was observed for the OLED of 1 (Figure 5). High luminous intensity is believed to be because of TADF emission of compound 1. Earlier reported acridone based compounds showed luminous intensity of $\sim 1000 \mathrm{Cd} / \mathrm{m}^{2}$ at a current density of $10 \mathrm{~mA} / \mathrm{cm}^{2}$ and $9800 \mathrm{Cd} / \mathrm{m}^{2}$ at $10 \mathrm{~mA} / \mathrm{cm}^{2}{ }^{16}$.

\section{Conclusions}

Acridone-amine derivative (1) was synthesized by substituting acyclic di-naphtyl amine on acridone. To study its prospects in TADF photophysical studies were carried out in detail. Compound $\mathbf{1}$ is fluorescent and emits in the green region $(550 \mathrm{~nm})$. Phosphorescence spectra was recorded at $77 \mathrm{~K}$ to find out the triplet energy level. The energy gap between the singlet and triplet state $\left(\Delta \mathrm{E}_{\mathrm{ST}}\right)$ is found to be $\sim 0.3 \mathrm{eV}$. Emission at $550 \mathrm{~nm}$ was also observed with a delay of $100 \mu \mathrm{s}$ which correspond to the delayed fluorescence in 1. The lifetime of TADF was found to be $176 \mu \mathrm{s}$ in a nitrogen-purged solvent. OLEDs based on 1 were fabricated and their intensity was found to be nearly $17,000 \mathrm{Cd} / \mathrm{m}^{2}$ at $25 \mathrm{~mA} / \mathrm{cm}^{2}$.

\section{Supplementary Information (SI)}

Spectra related to NMR, mass, absorption, emission and delayed lifetime studies, cyclic voltammogram is available at www.ias.ac.in/chemsci.

\section{Acknowledgement}

We thank Swati Dixit for her assistance in cyclic voltammetric studies. We thank Tata Institute of Fundamental Research, Mumbai for NMR and MALDI-TOF. We also thank the Radiation and Photochemistry Division, Bhabha Atomic Research Centre for TCSPC. NA and SB thank the Department of Science and Technology for partial financial support (EMR/2017/000805).

\section{References}

1. Sasabe H and Kido J 2011 Multifunctional Materials in High-Performance OLEDs: Challenges for Solid-State Lighting Chem. Mater. 23621

2. Ali F, Nayak P K, Periasamy N and Agarwal N 2017 Synthesis, photophysical, electrochemical and electroluminescence studies of red emitting phosphorescent Ir(III) heteroleptic complexes J. Chem. Sci. 1291391

3. Bhui P, Siddiqui Q T, Muneer M, Agarwal N and Bose S 2018 Deep blue organic light-emitting diodes of 1,8diaryl anthracene J. Chem. Sci. 130167

4. Forrest S R 2004 The path to ubiquitous and low-cost organic electronic appliances on plastic Nature $\mathbf{4 2 8} 911$

5. Nayak P K, Agarwal N, Ali F, Patankar M, Periasamy N and Narasimhan K L 2010 Blue and white light electroluminescence in a multilayer OLED using a new aluminium complex J. Chem. Sci. 122847

6. Siddiqui Q T, Bhui P, Muneer M, Chandrakumar K R S, Bose S and Agarwal N 2018 Nanoassembly of Dipolar Imidazoanthraquinone Derivatives Leading to Enhanced Hole Mobility J. Phys. Chem. C $\mathbf{1 2 2}$ 25804 
7. Xu R-P, Li Y-Q and Tanga J-X 2016 Recent advances in flexible organic light-emitting diodes J. Mater. Chem. C 49116

8. Yang Z, Mao Z, Xie Z, Zhang Y, Liu S, Zhao J, Xu J, Chi Z and Aldred M P 2017 Recent advances in organic thermally activated delayed fluorescence materials Chem. Soc. Rev. 46915

9. Forrest S R, Baldo M A, O'Brien D F, You Y, Shoustikov A, Sibley S and Thompson M E 1998 Highly Efficient Phosphorescent Emission from Organic Electroluminescent Devices Nature 395151

10. Méhes G, Nomura H, Zhang Q, Nakagawa $T$ and Adachi C 2012 Enhanced Electroluminescence Efficiency in a Spiro-acridine Derivative through Thermally Activated Delayed Fluorescence Angew. Chem. Int. Ed. 5111311

11. Nasu K, Nakagawa T, Nomura H, Lin C-J, Cheng C-H, Tseng M-R, Yasuda T and Adachi C 2013 A Highly Luminescent Spiro-Anthracenone-Based Organic Light-Emitting Diode Exhibiting Thermally Activated Delayed Fluorescence Chem. Commun. 4910385

12. Reineke S 2014 Organic Light-Emitting Diodes: Phosphorescence meets its Match Nat. Photonics 8269

13. Adachi C, Baldo M A, Thompson M E and Forrest S R 2001 Nearly $100 \%$ internal phosphorescence efficiency in an organic light-emitting device J. Appl. Phys. 90 5048

14. Cui L-S, Liu Y, Liu X-Y, Jiang Z-Q and Liao L-S 2015 Design and Synthesis of Pyrimidine-Based Iridium(III) Complexes with Horizontal Orientation for Orange and White Phosphorescent OLEDs ACS Appl. Mater. Interfaces 711007

15. Kaji H, Suzuki H, Fukushima T, Shizu K, Suzuki K, Kubo S, Komino T, Oiwa H, Suzuki F, Wakamiya A, Murata Y and Adachi C 2015 Purely Organic Electroluminescent Material Realizing 100\% Conversion from Electricity to Light Nat. Commun. 68476

16. Siddiqui Q T, Awasthi A A, Bhui P, Muneer M, Chandrakumar K R S, Bose S and Agarwal N 2019 Thermally Activated Delayed Fluorescence (Green) in Undoped Film and Exciplex Emission (Blue) in Acridone-Carbazole Derivatives for OLEDs J. Phys. Chem. C 1231003

17. Uoyama H, Goushi K, Shizu K, Nomura H and Adachi C 2012 Highly Efficient Organic Light-Emitting Diodes from Delayed Fluorescence Nature 492234

18. Wong M Y and Zysman-Colman E 2017 Purely Organic Thermally Activated Delayed Fluorescence Materials for Organic Light-Emitting Diodes $A d v$. Mater. 291605444

19. Zhao J, Chen X, Yang Z, Chi Z, Yang Z, Zhang Y, Xu J, Chi Z and Aldreda M P 2018 Highly-efficient fully non-doped white organic light-emitting diodes consisting entirely of thermally activated delayed fluorescence emitters J. Mater. Chem. C 63226

20. Grybauskaite-Kaminskiene G, Ivaniuk K, Bagdziunas G, Turyk P, Stakhira P, Baryshnikov G, Volyniuk D, Cherpak V, Minaev B, Hotra Z, Agrencg $\mathrm{H}$ and Grazulevicius J V 2018 Contribution of TADF and exciplex emission for efficient "warm-white" OLEDs J. Mater. Chem. C 61543

21. Zhang D, Duan L, Li C, Li Y, Li H, Zhang D and Qiu Y 2014 High-efficiency Fluorescent Organic LightEmitting Devices using Sensitizing Hosts with a Small Singlet-Triplet Exchange Energy. Adv. Mater. 265050

22. Penfold T J 2015 On Predicting the Excited-State Properties of Thermally Activated Delayed Fluorescence Emitters J. Phys. Chem. C 2413535

23. Mamada M, Inada $\mathrm{K}$, Komino $\mathrm{T}$, Potscavage $\mathrm{W} \mathbf{J} \mathrm{J}$, Nakanotani H and Adachi C 2017 Highly Efficient Thermally Activated Delayed Fluorescence from an Excited-State Intramolecular Proton Transfer System ACS Cent. Sci. 3769

24. Pander P, Swist A, Motyka R, Soloducho J, Dias F B and Data P 2018 Thermally Activated Delayed Fluorescence with a Narrow Emission Spectrum and Organic Room Temperature Phosphorescence by Controlling Spin-Orbit Coupling and Phosphorescence Lifetime of Metal-free Organic Molecules J. Mater. Chem. C 65434

25. Wang C, Zhang Z and Wang Y 2016 QuinacridoneBased $\pi$-Conjugated Electronic Materials J. Mater. Chem. C 49918

26. Wang Y, Zhu Y, Lin X, Yang Y, Zhang B, Zhan H, Xie Z and Cheng Y 2018 Efficient Non-doped Yellow OLEDs Based on Thermally Activated Delayed Fluorescence Conjugated Polymers with an Acridine/Carbazole Donor Backbone and Triphenyltriazine Acceptor Pendant J. Mater. Chem. C 6568

27. Sharma B K, Shaikh A M, Agarwal N and Kamble R M 2016 Synthesis, photophysical and electrochemical studies of acridone-amine based donor-acceptors for hole transport materials $R S C A d v$. 617129

28. Khopde S M, Priyadarsini K I, Palit D K and Mukherjee T 2000 Effect of solvent on the excitedstate photophysical properties of curcumin Photochem. Photobiol. 72625

29. Lakowicz J R 2006 Principle of Fluorescence Spectroscopy 3rd edn. (New York: Springer-Verlag)

30. Kappaun S, Slugove C and List E J W 2008 Phosphorescent Organic Light-Emitting Devices: Working Principle and Iridium Based Emitter Materials Int. J. Mol. Sci. 91527

31. Minaev B, Baryshnikov G and Agren H 2014 Principles of phosphorescent organic light emitting devices Phys. Chem. Chem. Phys. 161719

32. Masui K, Nakanotani H and Adachi C 2013 Analysis of Exciton Annihilation in High-efficiency Sky-Blue Organic Light-Emitting Diodes with Thermally Activated Delayed Fluorescence Org. Electron. 142721

33. Serevičius T, Nakagawa T, Kuo M-C, Cheng S-H, Wong K-T, Chang C-H, Kwong R C, Xia S and Adachi C 2013 Enhanced Electroluminescence based on Thermally Activated Delayed Fluorescence from a CarbazoleTriazine Derivative Phys. Chem. Chem. 1515850

34. Gritzner G and Kuta J 1984 Recommendations on Reporting Electrode Potentials in Nonaqueous Solvents J. Pure Appl. Chem. 56461 